\title{
The Menger and projective Menger properties of function spaces with the set-open topology
}

\author{
Alexander V. Osipov \\ Krasovskii Institute of Mathematics and Mechanics, Ural Federal University, \\ Ural State University of Economics, 620219, Yekaterinburg, Russia
}

\begin{abstract}
For a Tychonoff space $X$ and a family $\lambda$ of subsets of $X$, we denote by $C_{\lambda}(X)$ the space of all real-valued continuous functions on $X$ with the setopen topology. In this paper, we study the Menger and projective Menger properties of a Hausdorff space $C_{\lambda}(X)$. Our main results state that

if $\lambda$ is a $\pi$-network of $X$, then

(1) $C_{\lambda}(X)$ is Menger space, if and only, if $C_{\lambda}(X)$ is $\sigma$-compact, and, if $Y$ is a dense subset of $X$, then

(2) $C_{p}(Y \mid X)$ is projective Menger space, if and only, if $C_{p}(Y \mid X)$ is $\sigma$ pseudocompact.

Keywords: Menger, projective Menger, set-open topology, $\sigma$-compact, $\sigma$-pseudocompact, $\sigma$-bounded, basically disconnected space, function space 2010 MSC: 54C25, 54C35, 54C40, 54D20
\end{abstract}

\section{Introduction}

Throughout this paper $X$ will be a Tychonoff space. Let $\lambda$ be a family non-empty subsets of $X, C(X)$ the set of all continuous real-valued function on $X$. Denote by $C_{\lambda}(X)$ the set $C(X)$ is endowed with the $\lambda$-open topology. The elements of the standard subbases of the $\lambda$-open topology will be denoted as follows: $[F, U]=\{f \in C(X): f(F) \subseteq U\}$, where $F \in \lambda, U$ is an open subset of $\mathbb{R}$. Note that if $\lambda$ consists of all finite subsets of $X$ then the $\lambda$-open topology is equal to the topology of pointwise convergence, that

Email address: OAB@list.ru (Alexander V. Osipov) 
is $C_{\lambda}(X)=C_{p}(X)$. Denote be $C_{p}(Y \mid X)=\left\{h \in C_{p}(Y): h=\left.f\right|_{Y}\right.$ for $f \in C(X)\}$ for $Y \subset X$.

Recall that, if $X$ is a space and $\mathcal{P}$ a topological property, we say that $X$ is $\sigma$ - $\mathcal{P}$ if $X$ is the countable union of subspaces with the property $\mathcal{P}$.

So a space $X$ is called $\sigma$-compact ( $\sigma$-pseudocompact, $\sigma$-bounded), if $X=$ $\bigcup_{i=1}^{\infty} X_{i}$, where $X_{i}$ is a compact (pseudocompact, bounded) for every $i \in \mathbb{N}$. N.V. Velichko proved that $C_{p}(X)$ is $\sigma$-compact, if and only, if $X$ is finite. In [20], V.V. Tkachuk clarified when $C_{p}(X)$ is $\sigma$-pseudocompact and when $C_{p}(X)$ is $\sigma$-bounded, and considered similar questions for the space $C_{p}^{*}(X)$ of bounded continuous functions on $X$.

A space $X$ is said to be Menger [9] (or, [17]) if for every sequence $\left\{\mathcal{U}_{n}\right.$ : $n \in \omega\}$ of open covers of $X$, there are finite subfamilies $\mathcal{V}_{n} \subset \mathcal{U}_{n}$ such that $\bigcup\left\{\mathcal{V}_{n}: n \in \omega\right\}$ is a cover of $X$.

Every $\sigma$-compact space is Menger, and a Menger space is Lindelöf. The Menger property is closed hereditary, and it is preserved by continuous maps. It is well known that the Baire space $\mathbb{N}^{\mathbb{N}}$ (hence, $\mathbb{R}^{\omega}$ ) is not Menger.

In [2], A.V. Arhangel'skii proved that $C_{p}(X)$ is Menger, if and only, if $X$ is finite.

Let $\mathcal{P}$ be a topological property. A.V. Arhangel'skii calls $X$ projectively $\mathcal{P}$ if every second countable image of $X$ is $\mathcal{P}$. Arhangel'skii consider projective $\mathcal{P}$ for $\mathcal{P}=\sigma$-compact, analytic [3], and other properties.

Lj.D.R. Kočinac characterized the classical covering properties of Menger, Rothberger, Hurewicz and Gerlits-Nagy in term of continuous images in $\mathbb{R}^{\omega}$. The projective selection principles were introduced and first time considered in [11].

Every Menger space is projectively Menger. It is known (Theorem 2.2 in [11]) that a space is Menger, if and only, if it is Lindelöf and projectively Menger.

Characterizations of projectively Menger spaces $X$ in terms a selection principle restricted to countable covers by cozero sets are given in [5].

In [16], M. Sakai proved that $C_{p}(X)$ is projectively Menger, if and only, if $X$ is pseudocompact and $b$-discrete.

In this paper we study the Menger property of Hausdorff space $C_{\lambda}(X)$, and the projective Menger property of $C_{p}(Y \mid X)$ where $Y$ is dense subset of $X$. 


\section{Main definitions and notation}

Recall that a family $\lambda$ of non-empty subsets of a topological space $(X, \tau)$ is called a $\pi$-network for $X$ if for any nonempty open set $U \in \tau$ there exists $A \in \lambda$ such that $A \subset U$.

Throughout this paper, a family $\lambda$ of nonempty subsets of the set $X$ is a $\pi$-network. This condition is equivalent to the space $C_{\lambda}(X)$ being a Hausdorff space [12].

We will also need the following assertion [1], [4].

Proposition 2.1. If $\mathbb{I}_{\alpha}=\mathbb{I}=[0,1]$ for $\alpha \in A$ and $Y$ is a subspace of the Tychonoff cube $\mathbb{I}^{A}=\prod\left\{\mathbb{I}_{\alpha}: \alpha \in A\right\}$ which, whatever the countable set $B \subset A$, projects under the canonical projection $\pi_{B}: \mathbb{I}^{A} \mapsto \mathbb{I}^{B}$ onto the whole cube $\mathbb{I}^{B}=\prod\left\{\mathbb{I}_{\alpha}: \alpha \in B\right\}$ of $\mathbb{I}^{A}$, then $Y$ is pseudocompact.

Theorem 2.2. (Nokhrin [12]) For a Tychonoff space $X$ the following statements are equivalent:

1. $C_{\lambda}(X)$ is a $\sigma$-compact;

2. $X$ is a pseudocompact, $D(X)$ is a dense $C^{*}$-embedded set in $X$ and family $\lambda$ consists of all finite subsets of $D(X)$, where $D(X)$ is an isolated points of $X$.

The closure of a set $A$ will be denoted by $\bar{A}$ (or $\operatorname{cl}(A)$ ); the symbol $\varnothing$ stands for the empty set. As usual, $f(A)$ and $f^{-1}(A)$ are the image and the complete preimage of the set $A$ under the mapping $f$, respectively.

A subset $A$ of a space $X$ is said to be bounded in $X$ if for every continuous function $f: X \mapsto \mathbb{R}, f \mid A: A \mapsto \mathbb{R}$ is a bounded function. Every $\sigma$-bounded space is projectively Menger (Proposition 1.1 in [3] ).

\section{Main results}

In order to prove the main theorem we need to prove some statements that we call Lemmas, but note their self-importance.

Recall that a space $X$ is called basically disconnected ([8]), if every cozeroset has an open closure. Clearly, every basically disconnected (Tychonoff) space is zero-dimensional space.

Lemma 3.1. If $C_{\lambda}(X)$ is Menger, then $X$ is a basically disconnected space. 
Proof. Let $U \subseteq X$ be a cozero set in $X$. Claim that $\bar{U}=\operatorname{Int} \bar{U}$. Suppose that $\bar{U} \backslash \operatorname{Int} \bar{U} \neq \emptyset$. Since $U$ is a cozero set, there are open sets $U_{n}$ of $X$ such that for each $n \in \mathbb{N}, \overline{U_{n}} \subseteq U_{n+1}$ and $\bigcup_{n=1}^{\infty} U_{n}=U$. For each $n, m \in \mathbb{N}$, we put $Z_{n, m}=\left\{f \in C_{\lambda}(X,[0,1]): f \mid(X \backslash \operatorname{Int} \bar{U}) \equiv 0\right.$ and $\left.f\left(U_{n}\right) \subset\left[\frac{1}{2^{m}}, 1\right]\right\}$.

Note that $Z_{n, m}$ is closed subset of $C_{\lambda}(X)$ for each $n, m \in \mathbb{N}$. Let $h \notin Z_{n, m}$. If $x \in X \backslash \operatorname{Int} \bar{U}$ such that $h(x) \neq 0$. Since $\lambda$ is $\pi$-network of $X$, there is $A \in \lambda$ such that $A \subset h^{-1}\left(h(x)-\frac{|h(x)|}{2}, h(x)+\frac{|h(x)|}{2}\right) \bigcap \operatorname{Int}(X \backslash \operatorname{Int} \bar{U})$. Then $h \in\left[A,\left(h(x)-\frac{|h(x)|}{2}, h(x)+\frac{|h(x)|}{2}\right)\right]$ and $\left[A,\left(h(x)-\frac{\lfloor h(x) \mid}{2}, h(x)+\frac{|h(x)|}{2}\right)\right] \cap Z_{n, m}=$ $\emptyset$.

If $x \in U_{n}$ and $h(x) \notin\left[\frac{1}{2^{m}}, 1\right]$. Let $d=\frac{\operatorname{diam}\left(h(x),\left[\frac{1}{2^{m}}, 1\right]\right)}{2}$. Since $\lambda$ is a $\pi$ network of $X$, there is $A \in \lambda$ such that $A \subset h^{-1}\left((h(x)-d, h(x)+d) \cap U_{n}\right.$. Then $h \in[A,(h(x)-d, h(x)+d)]$ and $[A,(h(x)-d, h(x)+d)] \cap Z_{n, m}=\emptyset$.

Assume that $\bigcap\left\{Z_{n, m}: n \in \mathbb{N}\right\}=\emptyset$ for all $m \in \mathbb{N}$. Using the Menger property of $C_{\lambda}(X)$, we can take some $\varphi \in \mathbb{N}^{\mathbb{N}}$ such that $\bigcap\left\{Z_{\varphi(m), m}: m \in\right.$ $\mathbb{N}\}=\emptyset$. For each $m \in \mathbb{N}$, take any $g_{m} \in C_{\lambda}(X)$ satisfying $g_{m}(X \backslash \operatorname{Int}(\bar{U})) \equiv 0$ and $g_{m}\left(U_{\varphi(m)}\right)=\{1\}$. Let $g=\sum_{j=1}^{\infty} 2^{-j} g_{j}$. Then, $g \in C_{\lambda}(X)$ and $g(X \backslash$ $\operatorname{Int}(\bar{U})) \equiv 0$. Fix any $m \in \mathbb{N}, 1 \leq k \leq \varphi(m)$ and $x \in U_{k}$. Then we have $g(x)=\sum_{j=1}^{\infty} 2^{-j} g_{j}(x) \geq 2^{-m} g_{m}(x)=2^{-m}$.

Hence, $g \in \bigcap\left\{Z_{\varphi(m), m}: m \in \mathbb{N}\right\}$. This is a contradiction. Thus, there is some $m \in \mathbb{N}$ such that $\bigcap\left\{Z_{n, m}: n \in \mathbb{N}\right\} \neq \emptyset$. Let $p \in \bigcap\left\{Z_{n, m}: n \in \mathbb{N}\right\}$. Then $p(U) \subset\left[\frac{1}{2^{m}}, 1\right]$ and $p \mid(X \backslash \operatorname{Int} \bar{U}) \equiv 0$. It follows that $\bar{U} \backslash \operatorname{Int} \bar{U}=\emptyset$.

A subset $G \subset \omega^{\omega}$ is dominating if for every $f \in \omega^{\omega}$ there is a $g \in G$ such that $f(n) \leq g(n)$ for all but finitely many $n$.

Theorem 3.2. (Hurewicz [10]) A second countable space $X$ is Menger iff for every continuous mapping $f: X \mapsto \mathbb{R}^{\omega}, f(X)$ is not dominating.

"Second countable" can be extended to "Lindelöf":

Theorem 3.3. (Kočinac [11], Theorem 2.2) A Lindelöf space $X$ is Menger iff for every continuous mapping $f: X \mapsto \mathbb{R}^{\omega}, f(X)$ is not dominating.

Lemma 3.4. If $C_{\lambda}(X)$ is Menger. Then $X$ is pseudocompact.

Proof. Assume that $X$ is not pseudocompact and $f \in C(X)$ is not bounded function. Without loss of generality we can assume that $\mathbb{N} \subset f(X)$. For each $n \in \mathbb{N}$ we choose $A_{n} \in \lambda$ such that $A_{n} \subset f^{-1}\left(\left(n-\frac{1}{3}, n+\frac{1}{3}\right)\right)$. By 
Lemma [3.1, $F_{n}=\overline{f^{-1}\left(\left(n-\frac{1}{3}, n+\frac{1}{3}\right)\right)}$ is clopen set for each $n \in \mathbb{N}$. Let $K=\left\{f \in C(X): f \mid F_{n} \equiv s_{f, n}\right.$ for each $n \in \mathbb{N}$ and $\left.s_{f, n} \in \mathbb{R}\right\}$. Then $K$ is closed subset of $C_{\lambda}(X)$ and, hence, it is Menger. Fix $a_{n} \in A_{n}$ for every $n \in \mathbb{N}$. Note that $D=\left\{a_{n}: n \in \mathbb{N}\right\}$ is a $C$-embedded copy of $\mathbb{N}$ (3L (1) in [8]). So we have a continuous mapping $F: K \mapsto \mathbb{R}^{D}$ the space $K$ onto $\mathbb{R}^{D}$. But $F(K)=\mathbb{R}^{D}=\mathbb{R}^{\omega}$ is dominating, contrary to the Theorem 3.3 ,

Lemma 3.5. If $C_{\lambda}(X)$ is Menger, then $\mu=\{A \in \lambda: A$ is finite subset of $X\}$ is a $\pi$-network of $X$.

Proof. Assume that there exist an open set $U$ of $X$ such that $B \not \subset U$ for every $B \in \mu$. Fix a family $\left\{V_{n}: n \in \mathbb{N}\right\}$ of open subsets of $X$ such that $V_{n} \subset U$ for every $n \in \mathbb{N}$ and $V_{n^{\prime}} \bigcap V_{n^{\prime \prime}}=\emptyset$ for $n^{\prime} \neq n^{\prime \prime}$. Fix $x_{n} \in V_{n}$ and $\epsilon>0$. For every $f \in C_{\lambda}(X)$ and $n \in \mathbb{N}$ consider $B_{f, n} \in \lambda$ such that $B_{f, n} \subset f^{-1}\left(\left(f\left(x_{n}\right)-\right.\right.$ $\left.\left.\epsilon, f\left(x_{n}\right)+\epsilon\right)\right) \bigcap V_{n}$. Then $\mathcal{U}_{n}=\left\{\left[B_{f, n},\left(f\left(x_{n}\right)-\epsilon, f\left(x_{n}\right)+\epsilon\right)\right]: f \in C_{\lambda}(X)\right\}$ is an open cover of $C_{\lambda}(X)$ for every $n \in \mathbb{N}$. Using the Menger property of $C_{\lambda}(X)$, for sequence $\left\{\mathcal{U}_{n}: n \in \omega\right\}$ of open covers of $C_{\lambda}(X)$, there are finite subfamilies $\mathcal{S}_{n} \subset \mathcal{U}_{n}$ such that $\bigcup\left\{\mathcal{S}_{n}: n \in \omega\right\}$ is a cover of $C_{\lambda}(X)$. Let $\mathcal{S}_{n}=$ $\left\{\left[B_{f_{1}, n},\left(f_{1, n}\left(x_{n}\right)-\epsilon, f_{1, n}\left(x_{n}\right)+\epsilon\right)\right], \ldots,\left[B_{f_{k(n)}, n},\left(f_{k(n), n}\left(x_{n}\right)-\epsilon, f_{k(n), n}\left(x_{n}\right)+\epsilon\right)\right]\right\}$ for every $n \in \mathbb{N}$. Since $B_{f_{s}, n}$ is an infinite subset of $X$, we fix $z_{s, n} \in B_{f_{s}, n}$ for every $s \in \overline{1, k(n)}$ and $n \in \mathbb{N}$ such that $z_{s^{\prime}, n} \neq z_{s^{\prime \prime}, n}$ for $s^{\prime} \neq s^{\prime \prime}$. Let $Z=\left\{z_{s, n}: s \in \overline{1, k(n)}\right.$ and $\left.n \in \mathbb{N}\right\}$.

Define the function $q: Z \mapsto \mathbb{R}$ such that $q\left(z_{s, n}\right)=0$ if $0 \notin\left(f_{s, n}\left(x_{n}\right)-\right.$ $\left.\epsilon, f_{s, n}\left(x_{n}\right)+\epsilon\right)$, else $q\left(z_{s, n}\right)=2 \epsilon$ for $s \in \overline{1, k(n)}$ and $n \in \mathbb{N}$. By Lemma 3.1, $X$ is a basically disconnected space.

Recall that ( $14 \mathrm{~N}$ p.215 in [8]) every countable set in a basically disconnected space is $C^{*}$-embedded.

Hence, there is $t \in C_{\lambda}(X)$ such that $t \mid Z=q$. But $t \notin \bigcup\left\{\mathcal{S}_{n}: n \in \omega\right\}$. This is a contradiction.

Denote $D(X)$ a set of isolated points of $X$.

Lemma 3.6. If $C_{\lambda}(X)$ is Menger, then $D(X)$ is dense set in $X$.

Proof. Assume that there exist an open set $W \neq \emptyset$ such that $W \cap D(X)=\emptyset$. By Lemma 3.5, $\mu$ is $\pi$-network of $X$, hence, there is $A \in \mu$ such that $A \subset W$. Note that $X \backslash A$ is dense set in $X$. The constant zero function defined on 
$X$ is denoted by $f_{0}$. For every $f \in C(X) \backslash\left\{f_{0}\right\}$ there is $x_{f} \in X \backslash A$ such that $f\left(x_{f}\right) \neq 0$. For every $f \in C(X) \backslash\left\{f_{0}\right\}$, consider $B_{f} \in \mu$ such that $B_{f} \subset f^{-1}\left(\left(f\left(x_{f}\right)-\frac{\left|f\left(x_{f}\right)\right|}{2}, f\left(x_{f}\right)+\frac{\left|f\left(x_{f}\right)\right|}{2}\right)\right) \bigcap(X \backslash A)$. Let $\epsilon>0$. Then $\mathcal{V}=\left\{\left[B_{f},\left(f\left(x_{f}\right)-\frac{\left|f\left(x_{f}\right)\right|}{2}, f\left(x_{f}\right)+\frac{\left|f\left(x_{f}\right)\right|}{2}\right)\right]: f \in C(X) \backslash\left\{f_{0}\right\}\right\} \bigcup[A,(-\epsilon, \epsilon)]$ is an open cover of $C_{\lambda}(X)$. Since $C_{\lambda}(X)$ is Menger and, hence, $C_{\lambda}(X)$ is Lindelöf, there is a countable subcover $\mathcal{V}^{\prime}=\left\{\left[B_{f_{n}},\left(f_{n}\left(x_{f}\right)-\frac{\left|f_{n}\left(x_{f}\right)\right|}{2}, f_{n}\left(x_{f}\right)+\right.\right.\right.$ $\left.\left.\frac{\left|f_{n}\left(x_{f}\right)\right|}{2}\right)\right]$ : for $\left.n \in \mathbb{N}\right\} \bigcup[A,(-\epsilon, \epsilon)] \subset \mathcal{V}$ of $C_{\lambda}(X)$. Since $X$ is a basically disconnected space and every countable set in a basically disconnected space is $C^{*}$-embedded, there is $h \in C(X)$ such that $h \mid \bigcup_{n \in \mathbb{N}} B_{f_{n}} \equiv 0$ and $h(a)=\epsilon$ for some $a \in A$. Note that $h \notin \bigcup \mathcal{V}^{\prime}$, to contradiction.

Lemma 3.7. If $C_{\lambda}(X)$ is Menger, then $D(X)$ is $C^{*}$-embedded.

Proof. Let $f$ be a bounded continuous function from $D(X)$ into $\mathbb{R}$, and $F_{A}=$ $\{g \in C(X): g|A=f| A\}$ for $A \in D(X)^{\omega}$. Note that $F_{A}$ is closed subset of $C_{\lambda}(X)$ and, by Lemma 3.1, $F_{A} \neq \emptyset$. So $\xi=\left\{F_{A}: A \in D(X)^{\omega}\right\}$ is family of closed subspaces with the countable intersection property. Since $C_{\lambda}(X)$ is Menger, hence, it is Lindelöf, and every family of closed subspaces of with the countable intersection property has non-empty intersection. It follows that $\bigcap \xi \neq \emptyset$. We thus get that $\widetilde{f} \in \bigcap \xi$ such that $\widetilde{f} \in C(X)$ and $\widetilde{f} \mid D(X)=f$.

Proposition 3.8. Let $X=\mathbb{N}$ and let $\lambda=\{X\} \bigcup\{\{x\}: x \in X\}$. Then $C_{\lambda}^{*}(X)$ is not Menger.

Proof. Assume that $C_{\lambda}^{*}(X)$ is Menger. For every $i \in \mathbb{N}$ consider an open cover $\mathcal{V}_{i}=\left\{\left[\mathbb{N},\left(-2+\frac{1}{i+1}, 2-\frac{1}{i+1}\right)\right]\right\} \bigcup\left\{\left[x,\left(-\infty,-2+\frac{2 i+1}{2 i(i+1)}\right) \bigcup\left(2-\frac{2 i+1}{2 i(i+1)},+\infty\right)\right]:\right.$ $x \in X\}$ of $C_{\lambda}^{*}(X)$. Using the Menger property of $C_{\lambda}^{*}(X)$, for sequence $\left\{\mathcal{V}_{i}\right.$ : $i \in \mathbb{N}\}$ of open covers of $C_{\lambda}^{*}(X)$, there are finite subfamilies $\mathcal{S}_{i} \subset \mathcal{V}_{i}$ such that $\bigcup\left\{\mathcal{S}_{i}: i \in \mathbb{N}\right\}$ is a cover of $C_{\lambda}^{*}(X)$.

Without loss of generality we can assume that $\left[\mathbb{N},\left(-2+\frac{1}{i+1}, 2-\frac{1}{i+1}\right)\right] \in \mathcal{S}_{i}$ for each $i \in \mathbb{N}$.

By using induction, for each $i \in \mathbb{N}$, determine the values of the function $f$ at some points, depending on the $\mathcal{S}_{i}$, as follows:

for $i=1$ and

$\mathcal{S}_{1}=\left\{\left[\mathbb{N},\left(-2+\frac{1}{2}, 2-\frac{1}{2}\right)\right],\left[x_{1}^{1},\left(-\infty,-2+\frac{3}{4}\right) \bigcup\left(2-\frac{3}{4},+\infty\right)\right], \ldots,\left[x_{k}^{1},(-\infty,-2+\right.\right.$ $\left.\left.\left.\frac{3}{4}\right) \cup\left(2-\frac{3}{4},+\infty\right)\right]\right\}$, define 
$f\left(x_{n}^{1}\right)=0$ for $n \in \overline{1, k}$ and

$f\left(s_{1}\right)=p_{1}$ where $p_{1} \in\left[-2+\frac{5}{12}, 2-\frac{5}{12}\right] \backslash\left(-2+\frac{1}{2}, 2-\frac{1}{2}\right)$ for some $s_{1} \in$ $X \backslash\left\{x_{n}^{1}: n \in \overline{1, k}\right\}$. Denote $P_{1}=\bigcup_{n \in \overline{1, k}} x_{n}^{1} \bigcup s_{1}$.

for $i=m$

$\mathcal{S}_{m}=\left\{\left[\mathbb{N},\left(-2+\frac{1}{m+1}, 2-\frac{1}{m+1}\right)\right],\left[x_{1}^{m},\left(-\infty,-2+\frac{2 m+1}{2 m(m+1)}\right) \bigcup\left(2-\frac{2 m+1}{2 m(m+1)},+\infty\right)\right], \ldots\right.$

$\left.\ldots,\left[x_{k(m)}^{m},\left(-\infty,-2+\frac{2 m+1}{2 m(m+1)}\right) \bigcup\left(2-\frac{2 m+1}{2 m(m+1)},+\infty\right)\right]\right\}$, define

$f\left(x_{n}^{m}\right)=0$ where $x_{n}^{m} \notin P_{m-1}$ for $n \in \overline{1, k(m)}$ and

$f\left(s_{m}\right)=p_{m}$ where $p_{m} \in\left[-2+\frac{2(m+1)+1}{2(m+1)(m+2)}, 2-\frac{2(m+1)+1}{2(m+1)(m+2)}\right] \backslash\left(-2+\frac{1}{m+1}, 2-\right.$

$\left.\frac{1}{m+1}\right)$ for some $s_{m} \in X \backslash P_{m-1}$. Denote $P_{m}=\bigcup_{n \in 1, k(m)} x_{n}^{m} \bigcup s_{m} \bigcup P_{m-1}$ and $P=\bigcup_{m \in \mathbb{N}} P_{m}$

If $X \backslash P \neq \emptyset$, then let $f(x)=1$ for $x \in X \backslash P$.

By construction of $f, f \notin \mathcal{S}_{i}$ for every $i \in \mathbb{N}$, to contradiction.

Lemma 3.9. If $C_{\lambda}(X)$ is Menger, then each $A \in \lambda$ is finite subset of $D(X)$.

Proof. Suppose that $C_{\lambda}(X)$ is Menger, $\widetilde{\lambda}=\{A\} \bigcup\{\{x\}, x \in D(X)\}$ and $A \in \lambda$ is an infinite subset of $X$. Then $C_{\widetilde{\lambda}}(X)$ is Menger, too. Note that if $A$ is countable and $A \subset D(X)$, then we have a continuous mapping $g$ : $C_{\widetilde{\lambda}}(X) \mapsto C_{p \cup\{\mathbb{N}\}}^{*}(\mathbb{N})$. Hence, $C_{p \cup\{\mathbb{N}\}}^{*}(\mathbb{N})$ is Menger, contrary to Proposition 3.8 .

Let $V=(-1,1) \bigcup(\mathbb{R} \backslash[-4,4])$. Consider $\mathcal{U}=\{[A, V]\} \bigcup\left\{\left[x, \mathbb{R} \backslash\left[-\frac{2}{3}, \frac{2}{3}\right]\right]\right.$ : $x \in D(X)\}$. Since $D(X)$ is dense subset of $C_{\widetilde{\lambda}}(X)$ (Lemma 3.6), $\mathcal{U}$ is an open cover of $C_{\widetilde{\lambda}}(X)$ and, hence, there is a countable subcover $\mathcal{U}^{\prime} \subset \mathcal{U}$ of $C_{\widetilde{\lambda}}(X)$. Let $\mathcal{U}^{\prime}=\left\{[A, V],\left[x_{1}, \mathbb{R} \backslash\left[-\frac{2}{3}, \frac{2}{3}\right]\right], \ldots,\left[x_{n}, \mathbb{R} \backslash\left[-\frac{2}{3}, \frac{2}{3}\right]\right], \ldots\right\}$. Let $z \in A \backslash \bigcup_{n \in \mathbb{N}}\left\{x_{n}\right\}$ (note that either $z \in A \backslash D(X)$ or $A \subset D(X)$ and $|A|>\aleph_{0}$ ). Since every countable set in a basically disconnected space is $C^{*}$-embedded, there is $h \in C_{\widetilde{\lambda}}(X)$ such that $h \mid \bigcup_{n \in \mathbb{N}}\left\{x_{n}\right\}=0$ and $h(z)=2$. It follows that $h \notin \bigcup \mathcal{U}^{\prime}$, to contradiction. It follows that $A$ is finite subset of $D(X)$.

Theorem 3.10. Let $X$ be a Tychonoff space and let $\lambda$ be a $\pi$-network of $X$. Then a space $C_{\lambda}(X)$ is Menger, if and only if, $C_{\lambda}(X)$ is $\sigma$-compact.

Proof. By Lemma 3.4, $X$ is pseudocompact. By Lemmas 3.5 and 3.9, the family $\lambda$ consists of all finite subsets of $D(X)$, where $D(X)$ is an isolated 
points of $X$. By Lemma [3.7, $D(X)$ is a dense $C^{*}$-embedded set in $X$. It follows that $C_{\lambda}(X)$ is $\sigma$-compact (Theorem 2.2 ).

Various properties between $\sigma$-compactness and Menger are investigated in the papers [19, 6]. We can summarize the relationships between considered notions in ([19], see Figure 1), Theorems 3.10 and 2.2. Then we have the next

Theorem 3.11. For a Tychonoff space $X$ and a $\pi$-network $\lambda$ of $X$, the following statements are equivalent:

1. $C_{\lambda}(X)$ is $\sigma$-compact;

2. $C_{\lambda}(X)$ is Alster;

3. $(\mathrm{CH}) \mathrm{C}_{\lambda}(\mathrm{X})$ is productively Lindelöf;

4. "TWO wins $M$-game" for $C_{\lambda}(X)$;

5. $C_{\lambda}(X)$ is projectively $\sigma$-compact and Lindelöf;

6. $C_{\lambda}(X)$ is Hurewicz;

7. $C_{\lambda}(X)$ is Menger;

8. $X$ is a pseudocompact, $D(X)$ is a dense $C^{*}$-embedded set in $X$ and family $\lambda$ consists of all finite subsets of $D(X)$, where $D(X)$ is an isolated points of $X$.

\section{Projectively Menger space}

According to Tkačuk [20], a space $X$ said to be $b$-discrete if every countable subset of $X$ is closed (equivalently, closed and discrete) and $C^{*}$-embedded in $X$.

Lemma 4.1. (Lemma 2.1 in [16]) The following are equivalent for a space $X$ :

1. $X$ is b-discrete;

2. For any disjoint countable subsets $A$ and $B$ in $X$, there are disjoint zero-sets $Z_{A}$ and $Z_{B}$ in $X$ such that $A \subset Z_{A}$ and $B \subset Z_{B}$;

3. For any disjoint countably subsets $A$ and $B$ in $X$ such that $A$ is closed in $X$, there are disjoint zero-sets $Z_{A}$ and $Z_{B}$ in $X$ such that $A \subset Z_{A}$ and $B \subset Z_{B}$. 
Definition 4.2. For $A \subset X$, a space $X$ will be called $b_{A}$-discrete if every countable subset of $A$ is closed in $A$ and $C^{*}$-embedded in $X$.

Lemma 4.3. The following are equivalent for a space $X$ and $A \subset X$ :

1. $X$ is $b_{A}$-discrete;

2. For any disjoint countable subsets $D$ and $B$ in $A$, there are disjoint zero-sets $Z_{D}$ and $Z_{B}$ in $X$ such that $D \subset Z_{D}$ and $B \subset Z_{B}$;

3. For any disjoint countably subsets $D$ and $B$ in $A$ such that $D$ is closed in $A$, there are disjoint zero-sets $Z_{A}$ and $Z_{B}$ in $X$ such that $D \subset Z_{D}$ and $B \subset Z_{B}$.

Similarly to the proof of implication $\left(C_{p}(X, \mathbb{I})\right.$ is projectively Menger $\Rightarrow$ $X$ is $b$-discrete) of Theorem 2.4 in [16], we claim the next

Lemma 4.4. Let $C_{\lambda}(X)$ be a projectively Menger space, then $X$ is $b_{A}$-discrete where $A=\bigcup \lambda$.

Proof. Let $C_{\lambda}(X)$ be a projectively Menger. We show the statement (3) in Lemma 4.3. Let $D$ and $B$ be a disjoint countable subsets in $A$ such that $D$ is closed in $A$. Let $B=\left\{b_{n}: n \in \mathbb{N}\right\}$, and let $B_{n}=\left\{b_{1}, \ldots, b_{n}\right\}$.

For each $n, m \in \mathbb{N}$, we put $Z_{n, m}=\left\{f \in C_{\lambda}(X): f(D)=\{0\}\right.$ and $\left.f\left(B_{m}\right) \subset\left[\frac{1}{2^{n}}, 1\right]\right\}$. Since $D$ and $B_{m}$ are countable and $\lambda$ is a $\pi$-network of $X$, each $Z_{n, m}$ is a zero-set in $C_{\lambda}(X)$. Assume that $\bigcap\left\{Z_{n, m}: m \in \mathbb{N}\right\}=\emptyset$ for all $n \in \mathbb{N}$. Using the projective Menger property of $C_{\lambda}(X)$, Theorem 6 in [5], we can take some $\varphi \in \mathbb{N}^{\mathbb{N}}$ such that $\bigcap\left\{Z_{n, \varphi(n)}: n \in \mathbb{N}\right\}=\emptyset$. For each $n \in \mathbb{N}$, take any $g_{n} \in C_{\lambda}(X)$ satisfying $g_{n}(D)=\{0\}$ and $g_{n}\left(B_{\varphi(n)}=\{1\}\right.$. Let $g=\sum_{j=1}^{\infty} 2^{-j} g_{j}$. Then, $g \in C_{\lambda}(X)$ and $g(D) \equiv 0$. Fix any $n \in \mathbb{N}$, $1 \leq k \leq \varphi(m)$. Then we have

$g\left(b_{k}\right)=\sum_{j=1}^{\infty} 2^{-j} g_{j}\left(b_{k}\right) \geq 2^{-n} g_{n}\left(b_{k}\right)=2^{-n}$.

Hence, $g \in \bigcap\left\{Z_{n, \varphi(n)}: n \in \mathbb{N}\right\}$. This is a contradiction. Thus, there is some $n \in \mathbb{N}$ such that $\bigcap\left\{Z_{n, m}: m \in \mathbb{N}\right\} \neq \emptyset$. Let $h \in \bigcap\left\{Z_{n, m}: m \in \mathbb{N}\right\}$. Then $D \subset Z_{A}=h^{-1}(0)$ and $B \subset Z_{B}=h^{-1}\left(\left[\frac{1}{2^{n}}, 1\right]\right)$.

Theorem 4.5. Let $X$ be a Tychonoff space and let $Y$ be a dense subset of $X$. Then the following statements are equivalent:

1. $C_{p}(Y \mid X)$ is projectively Menger; 
2. $C_{p}(Y \mid X)$ is $\sigma$-bounded;

3. $C_{p}(Y \mid X)$ is $\sigma$-pseudocompact;

4. $X$ is pseudocompact and $b_{Y}$-discrete.

Proof. Note that $C_{p}(Y \mid X)$ is homeomorphic to $C_{\lambda}(X)$ for $\lambda=[Y]^{<\omega}$.

$(1) \Rightarrow(4)$. By Lemma 4.4, $X$ is $b_{Y}$-discrete. Assume that $X$ is not pseudocompact and $f \in C(X)$ is not bounded function. Without loss of generality we can assume that $\mathbb{N} \subset f(X)$. For each $n \in \mathbb{N}$ we choose $a_{n} \in Y$ such that $a_{n} \in f^{-1}\left(\left(n-\frac{1}{3}, n+\frac{1}{3}\right)\right)$. Note that $D=\left\{a_{n}: n \in \mathbb{N}\right\}$ is a $C$-embedded copy of $\mathbb{N}$ (3L (1) in [8]). So we have a continuous mapping $F: C_{p}(Y \mid X) \mapsto \mathbb{R}^{D}$ the Menger space $C_{p}(Y \mid X)$ onto $\mathbb{R}^{D}$. But $F\left(C_{p}(Y \mid X)\right)=$ $\mathbb{R}^{D}=\mathbb{R}^{\omega}$ is dominating, contrary to the Theorem 3.3 .

$(4) \Rightarrow(3)$. Since $C_{p}(Y \mid X, \mathbb{I})$ is a dense subset of $\mathbb{I}^{Y}$ and $X$ is $b_{Y}$-discrete, by Proposition 2.1, $C_{p}(Y \mid X, \mathbb{I})$ is pseudocompact. Hence, $C_{p}(Y \mid X)$ is $\sigma$ pseudocompact.

Note that every $\sigma$-pseudocompact space is $\sigma$-bounded, and every $\sigma$-bounded space is projectively Menger (Proposition 1.1 in [3]).

\section{Examples}

Using Theorem 3.10 and Theorem 4.5, we can construct example of projective Menger topological group $C_{\lambda}(X)$ such that it is not Menger.

Note that if $\lambda=[\bigcup \lambda]^{<\omega}$, then $C_{\lambda}(X)$ is a topological group (locally convex topological vector space, topological algebra) ([14], [15]).

Example 5.1. (Example 1 in [13]) Let $T$ be a P-space without isolated points, $X=\beta(T)$ and let $\lambda$ be a family of all finite subsets of $T$. Then $C_{\lambda}(X)$ is $\sigma$-countably compact (Theorem 1.2 in [13]), hence, the topological group $C_{\lambda}(X)$ is projective Menger. But the space $X$ does not contain isolated points, hence, $C_{\lambda}(X)$ is not Menger.

Example 5.2. (Example 2 in [13]) Let $D$ be an uncountable discrete space and $\lambda=D^{<\omega}$. Consider $F=\beta(D) \backslash \bigcup\{\bar{S}: S \subset D$, and $S$ countable $\}$. Denote by $b(D)$ a quotient space obtained from $\beta(D)$ by identifying the set $F$ with the point $\{F\}$. Then the topological group $C_{\lambda}(b(D))$ is projective Menger ( $\sigma$-countably compact), but is not Menger.

Example 5.3. ([18]) D.B.Shahmatov has constructed for an arbitrary cardinal $\tau \geq 2^{\aleph_{0}}$ an everywhere dense pseudocompact space $X_{\tau}$ in $\mathbb{I}^{\tau}$ such that 
$X_{\tau}$ is a b-discrete. Hence, the topological group $C_{p}\left(X_{\tau}\right)$ is projective Menger ( $\sigma$-pseudocompact and is not $\sigma$-countably compact), but is not Menger for an arbitrary cardinal $\tau \geq 2^{\aleph_{0}}$.

Remark 5.4. By Theorems 2.2 and 3.10, if $X$ is compact, $\lambda$ is a $\pi$-network of $X$ and $C_{\lambda}(X)$ is Menger, then $X$ is homeomorphic to $\beta(D)$, where $\beta(D)$ is Stone- $\breve{C}$ ech compactification of a discrete space $D$, and $\lambda=[D]^{<\omega}$.

\section{References}

[1] A.V. Arhangel'skii, Continuous maps, factorization theorems, and function spaces, Trudy Moskovsk. Mat. Obshch., 47, (1984), 3-21.

[2] A.V. Arhangel'skii, Hurewicz spaces, analytic sets and fan tightness of function spaces, Sov. Math. Dokl., 33, (1986), 396-399.

[3] A.V. Arhangel'skii, Projective $\sigma$-compactness, $\omega_{1}$-caliber, and $C_{p}$-spaces, Topology and its Applications, 157, (2000), 874-893.

[4] A.V. Arhangel'skii, V.I. Ponomarev, Fundamentals of general topology: problems and excercises, Reidel, 1984. (Translated from the Russian.)

[5] M. Bonanzinga, F. Cammaroto, M. Matveev, Projective versions of selection principles, Topology and its Applications, 157, (2010), 874-893.

[6] H. Duanmu, F.D. Tall, L. Zdomskyy, Productively Lindelöf and indestructibly Lindelöf spaces, Topology and its Applications 160:18 (2013), 2443-2453.

[7] R. Engelking, General Topology, PWN, Warsaw, (1977); Mir, Moscow, (1986).

[8] L. Gillman, M. Jerison, Rings of continuous functions, The University Series in Higher Mathematics. Princeton, New Jersey: D. Van Nostrand Co., Inc., 1960. 300 p.

[9] W. Hurewicz, Über eine verallgemeinerung des Borelschen Theorems, Math. Z. 24 (1925) 401-421.

[10] W. Hurewicz, Über folger stetiger funktionen, Fund. Math. 9 (1927) 193204. 
[11] Lj.D.R. Kočinac, Selection principles and continuous images, Cubo Math.J. 8 (2) (2006) 23-31.

[12] S.E. Nokhrin, Some properties of set-open topologies, Jurnal of Mathematical Sciences, issue 144, n 3, (2007) 4123-4151.

[13] A.V. Osipov, E.G. Pytkeev, On the $\sigma$-countable compactness of spaces of continuous functions with the set-open topology, Proceedings of the Steklov Institute of Mathematics, issue 285, n. S1, (2014) 153-162.

[14] A.V. Osipov, Topological-algebraic properties of function spaces with setopen topologies, Topology and its Applications, issue 3, n. 159, (2012) 800-805.

[15] A.V. Osipov, Group structures of a function spaces with the set-open topology, Sib. Ėlektron. Mat. Izv., 14, (2017) 1440-1446.

[16] M. Sakai, The projective Menger property and an embedding of $S_{\omega}$ into function spaces, Topology and its Applications, Vol. 220 (2017) 118-130.

[17] M. Sakai, M. Scheepers, The combinatorics of open covers in: K.P. Hart, J. van Mill, P.Simon (Eds.), Recent Progress in General Topology III, Atlantic Press, 2014, pp. 751-799.

[18] D.B. Shahmatov, A pseudocompact Tychonoff space all countable subsets of which are closed and $C^{*}$-embedded, Topology and its Applications, 22:2, (1986), 139-144.

[19] F.D. Tall, Productively Lindelöff spaces may all be D, Canadian Mathematical Bulletin 56:1 (2013), 203-212.

[20] V.V. Tkačuk, The spaces $C_{p}(X)$ : decomposition into a countable union of bounded subspaces and completeness properties, Topology and its Applications, n 22, (1986), 241-253. 